\title{
Spontaneous Polarization and Dielectric Relaxation Dynamics of Two Novel Diastereomeric Ferroelectric Liquid Crystals
}

\begin{abstract}
Lei-Ching Huang and ChaO-Ming Fu*
Department of Physics, National University of Taiwan, Taipei 106, Taiwan

(Received March 15, 2015; revised version September 20, 2015; in final form November 29, 2015)

This study reports on the spontaneous polarization and molecular dynamics of two diastereomeric ferroelectric liquid crystals with a phenyl core ring but with different types of diastereomeric structures. The magnitudes of spontaneous polarization, measured via an Automated LC Property Tester, were similar for the two ferroelectric liquid crystals. The complex dielectric spectra, measured via an impedance analyzer, indicated the existence of the Goldstone mode in the ferroelectric $\left(\mathrm{SmC}^{*}\right)$ phase for the two ferroelectric liquid crystals. Moreover, a novel ferrielectric-like $\left(\mathrm{SmC} \gamma^{*}\right.$-like) phase was observed at certain temperatures. The complex dielectric spectra of the two ferroelectric liquid crystals were optimally analyzed by a theoretical model composed of the Debye model and the Cole-Cole model. The physical parameters such as relaxation frequency and dielectric strength were determined by a dielectric relaxation study, and the obtained parameters varied in accordance with phase transition sequence. The experimental results of dielectric spectra and spontaneous polarization are explained in the discussion of the mesomorphic properties related to the ferroelectric liquid crystal molecular structure.
\end{abstract}

DOI: 10.12693/APhysPolA.129.97

PACS: 77.84.Nh, 77.22.Gm

\section{Introduction}

The application of liquid crystals to optical devices has been widespread for a few decades. Of the entire class of liquid crystals, ferroelectric liquid crystals (FLCs) have attracted interest not only in fundamental research but also for their technological application [1-4]. The spontaneous polarization $(\mathrm{Ps})$, which is dramatically affected by the structure of the FLC compounds [4-6], is an important factor in their technological applications.

Dielectric spectroscopy of the FLC molecules plays an important role in their application for electric-optical devices, since measurement of the dielectric response enables one to depict the molecular dynamics of FLCs. Dielectric relaxation in the chiral smectic $\mathrm{C}\left(\mathrm{SmC}^{*}\right)$ phase occurs as a result of collective dielectric processes related to director reorientational motion and the molecular reorientation processes associated with the polarization of the molecules. The collective dielectric processes are mainly attributed to the Goldstone mode (GM) and the soft mode (SM) [7-13]. Moreover, for FLC compounds with a high degree of Ps, the domain mode (DM) exists. Additionally, several studies have proposed a new domain structure $[9,11,14,15]$. Furthermore, at certain temperatures, the FLCs exhibit the $L$-mode and the $S$-mode [16-18].

In addition, dielectric spectra can be analyzed to distinguish liquid crystal phases. For example, Hiller et al. have investigated the ferrielectric phase $\left(\mathrm{SmC} \gamma^{*}\right)$ that contributes to the dielectric spectra [19], in which a loss peak can be observed at a frequency of about one

*corresponding author; e-mail: chaomingfu@phys.ntu.edu.tw decade below the characteristic frequency of the GM during phase transition from the ferroelectric to the ferrielectric phase. The observed loss peak is attributed to the helical fluctuation mode in the $\mathrm{SmC} \gamma^{*}$ phase; in other words, it is caused by some kind of ferrielectric Goldstone mode relaxation.

This study describes the temperature dependence of spontaneous polarization and the dielectric spectra of two diastereomeric ferroelectric liquid crystals with a phenyl core structure. The experimental results have demonstrated that the values of spontaneous polarization were similar for the two FLCs. Moreover, the dielectric spectra of the two samples exhibited the GM in the ferroelectric phase $\left(\mathrm{SmC}^{*}\right)$. Moreover, it is to be noted that the $\mathrm{SmC} \gamma^{*}$-like phase occurred at certain temperatures. In order to explore the molecular dynamics that contributed to the dielectric behavior of the two FLC samples, the dielectric spectra were analyzed by means of a theoretical model. By means of the comparison of the experimental results of this study with those of our previous investigation published in Ref. [20], a better understanding of the dielectric characteristic correlating to the molecular structure of the FLC compounds is provided.

\section{Experiments}

In Fig. 1, the molecular structures of the two FLC samples used for this study are depicted. Please refer to reports [21-23] for the details on FLC synthesis. The structural difference between the two FLC compounds was in the diastereomeric molecules. The diastereomeric structure of sample A was of $(S, S)$-configuration. However, the diastereomeric structure of sample $\mathrm{B}$ was of $(S, R)$ configuration. The liquid crystal phase and phase transition temperatures of the FLC samples, being identified 


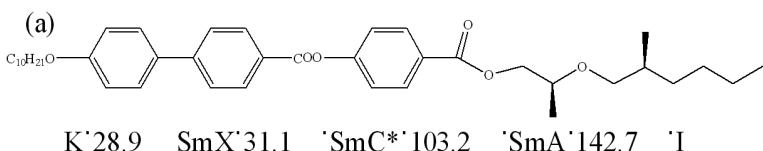

(b)

K'28.9 SmX'31.1 'SmC*'103.2 'SmA'142.7 'I

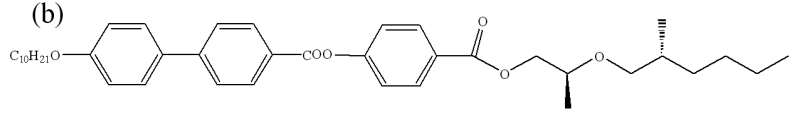

K'28.7 $\quad \operatorname{SmX}^{*} 65.0 \quad{ }^{\circ} \mathrm{SmC}^{*} \cdot 112.2 \quad{ }^{\circ} \mathrm{SmA}^{*} 143.3 \quad$ I

Fig. 1. Molecular structure and phase sequence of the two ferroelectric liquid crystal samples: (a) sample A $4-\{2(S)-[2(S)$-methylhexoxy]propylcarbonyl $\}$ phenyl-

4-(4-n- decoxy)phenybenzoate, (b) sample B 4-

$\{2(S)-[2(R)$ - methylhexoxy]propylcarbonyl $\}$ phenyl-4-

(4-n-decoxy)phenybenzoate.

by differential scanning calorimetry (DSC) and polarizing microscopy, are indicated in Fig. 1. Herein, the SmX is an unidentified liquid crystal phase from the investigations of DSC and polarizing microscope. As shown in Fig. 1, the temperature ranges of the liquid crystal phase for the two samples were similar (about $114^{\circ} \mathrm{C}$ ).

Sandwich-type sample cell was used for the spontaneous polarization and dielectric measurement. The commercial cells having $4 \mu \mathrm{m}$ thickness were coated with indium tin oxide (ITO) transparent electrodes with unidirectionally rubbed polyimide layers. The FLC samples were filled by means of capillarity.

The spontaneous polarization was measured through the polarization current reversal peak method [24] with a triangular wave of amplitude of $20 \mathrm{~V}_{\mathrm{rms}}$ and a frequency of $100 \mathrm{~Hz}$. The dielectric measurement of the sample was carried out by an HP4284A LCR meter with an ac electric field of $0.01 \mathrm{~V} \mu \mathrm{m}^{-1}$ within the frequency range of $20 \mathrm{~Hz}-1 \mathrm{MHz}$. The spontaneous polarization and dielectric spectrum were measured for the two FLC samples by cooling them from the liquid phase to the crystal phase.

The dielectric response of FLCs shows complex behavior as a function of both frequency and temperature. The experimental dielectric spectra were further analyzed with the Debye model and the Cole-Cole model, as expressed in Eq. (1)

$$
\begin{aligned}
\varepsilon^{*} & =\varepsilon_{\infty}+\frac{\Delta \varepsilon_{j}}{1+\left(\mathrm{i} \frac{f}{f_{r_{j}}}\right)}+\frac{\Delta \varepsilon_{j}}{1+\left(\mathrm{i} \frac{f}{f_{r_{j}}}\right)^{1-\alpha_{j}}} \\
& +\frac{1}{\left(\mathrm{i} 2 \pi f \tau_{c}\right)^{\gamma}},
\end{aligned}
$$

to depict the microscopic molecular dynamics of the two FLC samples. $\varepsilon_{\infty}$ in Eq. (1) is the real permittivity for high frequencies compared to $f_{\max }$. The second and third terms represent the Debye model and the Cole-Cole mode functions [25], respectively $\Delta \varepsilon_{j}, f_{r_{j}}$, and $\alpha_{j}$ are the respective dielectric strength, relaxation frequency and dispersion parameter of the $j$-th mode. In our experiment, the ITO electrodes of FLC cells were covered with a buffed polymer layer that possessed a strong rubbing property, causing a charge-accumulation effect. Thus, the Maxwell-Wagner effect [26], which is represented by the last term of Eq. (1), should contribute to dielectric relaxation in the low-frequency region $[27,28]$. The relaxation time $\tau_{c}$ in Eq. (1) depends not only on conductivity but also on the geometry of the sample. In Eq. (1), $\gamma$ is defined as $1-\alpha$, where $\alpha$ represents the dispersion parameter of the Maxwell-Wagner effect. All these parameters were obtained by the optimal fitting of experimental data into Eq. (1).

\section{Results and discussion}

Figure 2 shows the temperature dependence of spontaneous polarization of the two FLC samples in this study, measured through the triangular wave method. The spontaneous polarization was measured for the two samples by cooling them from the liquid phase to the crystal phase.
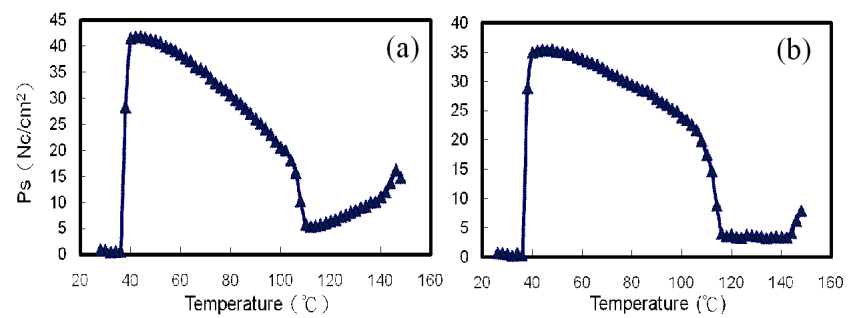

Fig. 2. Spontaneous polarization from liquid phase to crystal phase of (a) sample A and (b) sample B.

As seen in Fig. 2, the Ps values were similar for the two samples, both having a phenyl ring core structure. Mentioning the Ps dependence on temperature while cooling down to the $\mathrm{SmC}^{*}$ phase, the Ps value of sample A $((S, S)$-configuration) varied in a more intense way than that of sample $\mathrm{B}((S, R)$-configuration). This difference between the two samples was attributed to the diastereomeric structure of the FLC molecules.
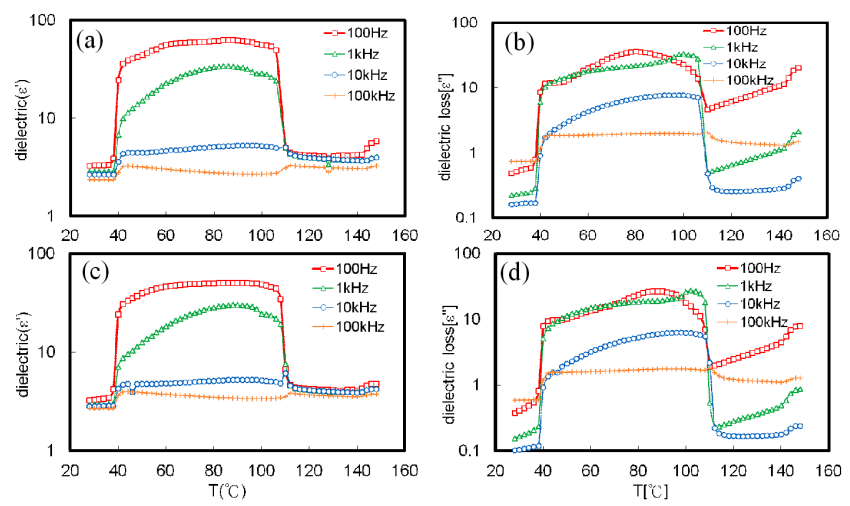

Fig. 3. The complex dielectric $\varepsilon^{*}$ in function of temperature $(T)$ at some frequencies of the two samples: the real part $\varepsilon^{\prime}$ of (a) sample A and (c) sample B; the imaginary part $\varepsilon^{\prime \prime}$ of (b) sample A and (d) sample B.

In order to get an understanding of the molecular dynamics of the two FLC samples, complex dielectric spectra dependent on temperature were measured from the liquid phase to the crystal phase for the two samples. 
Figure 3 shows the real part $\varepsilon^{\prime}$ and imaginary part $\varepsilon^{\prime \prime}$ of complex dielectric $\varepsilon^{*}$ in function of temperature $(T)$ for the two samples at four frequencies.
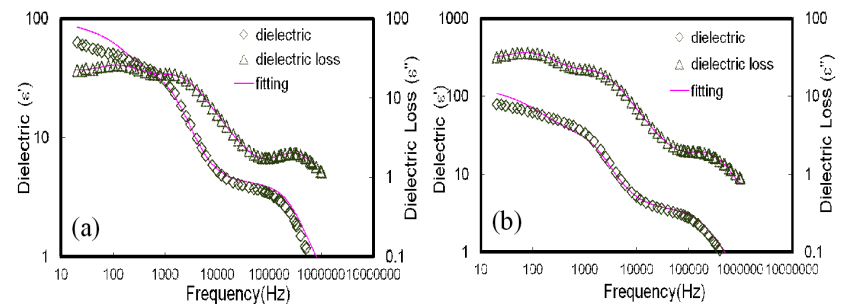

Fig. 4. Analyzed results of experimental data with the theoretical model according to Eq. (1) for the two FLCs at certain temperatures: (a) sample $\mathrm{A}$ at $82{ }^{\circ} \mathrm{C}$ and (b) sample B at $92{ }^{\circ} \mathrm{C}$ (open diamonds: experimental data of $\varepsilon^{\prime}(f)$. Open triangles: experimental data of $\varepsilon^{\prime \prime}(f)$. The solid line is the optimal curve found using the theoretical model).

As seen in Fig. 3, the values of $\varepsilon^{*}$ at four frequencies show variations with temperature that correspond to the phase sequences of the two samples (shown in Fig. 1). It is worth noting that significant variation was exhibited for $\varepsilon^{\prime}$ between $100 \mathrm{~Hz}$ and $1 \mathrm{kHz}$ within the temperature range of about $40^{\circ} \mathrm{C}$ to $110^{\circ} \mathrm{C}$, which corresponded to the $\mathrm{SmC}^{*}$ phase region of the two samples. Moreover, it is to be noted that in Fig. 3b and d, the values of $\varepsilon^{\prime \prime}$ in lower frequencies (below $1 \mathrm{kHz}$ ) in the liquid phase and the SmA phase decreased with the decrease of the temperatures, which corresponded to the variation of spontaneous polarization of the two samples in Fig. 2.

Figure 4 shows the frequency dependence of the dielectric permittivity $\varepsilon^{\prime}$ (primary axis) and dielectric loss $\varepsilon^{\prime \prime}$ (secondary axis) of the two samples at certain temperatures for the $\mathrm{SmC}^{*}$ phase, which had been identified by DSC and polarizing microscopy. It is to be noted that there were three relaxation modes in the dielectric spectra of Fig. 4, corresponding to the variation in Fig. 3. This specific characteristic of three relaxation modes was unexpected for the $\mathrm{SmC}^{*}$ phase, which usually only displays two relaxation modes. Comparing with the experimental data for four FLC samples which have been published in Ref. [20], although the molecular structures of the two FLC samples in this study were similar to those of the four samples in Ref. [20], the existence of three modes in Fig. 4 for the two samples of this study was distinct from the two modes in the dielectric spectra for the four samples reported in Ref. [20]. In order to obtain the quantitative information of molecular dynamics, a theoretical model composed of one Debye model, two Cole-Cole models and the Maxwell-Wagner effect was utilized to analyze the experimental results with three relaxation modes. The solid line in Fig. 4 represents the optimal results of the dielectric spectra for the two samples analyzed by using Eq. (1). As shown in Fig. 4a and $\mathrm{b}$, the experimental data agreed well with the theoretical model. The analyzed results depict that three relaxation modes contributed to the dielectric spectra.
In Fig. 4, the medium-frequency relaxation, whose characteristic frequency is about $1 \mathrm{kHz}$, is apparently contributed by the Goldstone mode (GM) [1]. Since the dielectric spectra in Fig. 4 was measured at 20 degrees below the $\mathrm{SmA}^{*}-\mathrm{SmC}^{*}$ phase transition temperature, there should be no soft mode (SM). As pointed out by Ref. [29], the relaxation mode associated to ITO electrodes will contribute to the high-frequency relaxation. Since the dielectric measurement of FLC samples in our experiment was carried out with ITO transparent electrodes, the high-frequency relaxation in Fig. 4 should be contributed by ITO pseudo-relaxation.

Since the SM was overlapped by the ITO pseudorelaxation, the information of SM has been masked by cell properties. In addition, the low intensity of highfrequency permittivity led the researchers to assume that the high-frequency relaxation was predominantly a result of ITO pseudo-relaxation instead of the liquid crystal properties [29]. The discussion below will focus on the relaxations in the low-frequency and the medium-frequency regions.

Further details of molecular dynamics for the lowfrequency and the medium-frequency relaxations for the two FLC samples can be seen in Fig. 5. Figure 5 shows the physical parameters, such as dielectric strength, relaxation frequency, and dispersion parameters which were obtained by analysis of experimental dielectric spectra through Eq. (1).

From analysis of the dielectric spectra of the two FLC samples, the optimal fitted parameters were obtained. Figure 5 shows the extracted fitted parameters, such as dielectric strength $\Delta \varepsilon_{j}$, relaxation frequency $f_{r_{j}}$, and dispersion parameter $\alpha_{j}$, where $j=1$ for the mode at medium frequencies (about $1 \mathrm{kHz}$ ), and $j=2$ represents the mode at low frequencies $(\approx 1-400 \mathrm{~Hz})$. According to the experimental results, the low-frequency relaxation mode occurred in the dielectric spectra for sample A at $48^{\circ} \mathrm{C}$ to $96^{\circ} \mathrm{C}$ and sample B at $39^{\circ} \mathrm{C}$ to $98^{\circ} \mathrm{C}$. As previously mentioned regarding the contributing factor of the third relaxation, according to Ref. [19], a helical fluctuation mode in the $\mathrm{SmC} \gamma^{*}$ phase contributes a loss peak with high-intensity relaxation at the frequency of about one decade below the characteristic frequency of the GM. In this study, the low-frequency relaxation occurred at about $100 \mathrm{~Hz}$, that is, one order lower than that of the GM (about $1 \mathrm{kHz}$ ). Therefore, in this study, the $\mathrm{SmC} \gamma^{*}$-like phase, caused by the ferrielectric Goldstone mode, was observed in the two FLC samples. Comparing to the experimental results and the molecular structures of the four FLC samples reported in Ref. [20], the existence of the $\mathrm{SmC} \gamma^{*}$-like phase was attributed to the phenyl core ring of the two FLC samples.

As mentioned above, the $\mathrm{SmC} \gamma^{*}$-like phase was observed from the results of the dielectric measurement. However, the $\mathrm{SmC} \gamma^{*}$ phase was not detected in the phase transition of the two FLC samples in Fig. 1, which was obtained from the investigations of DSC and polarizing microscope. According to Ref. [30], this situation could 
be interpreted by two reasons. First of all, the phase transition $\mathrm{SmC}^{*}-\mathrm{SmC}^{*}$ is low-energetic, so it is not easy to be detected by using standard DSC measurement. Second, the appearance of ferrielectric phase strongly depends on the cell thickness. Furthermore, in our samples, as shown in Fig. 1, there was an unidentified liquid crystal phase which was tentatively named as SmX. Therefore, the existence of any kind of liquid crystal phase in this temperature region cannot be excluded.
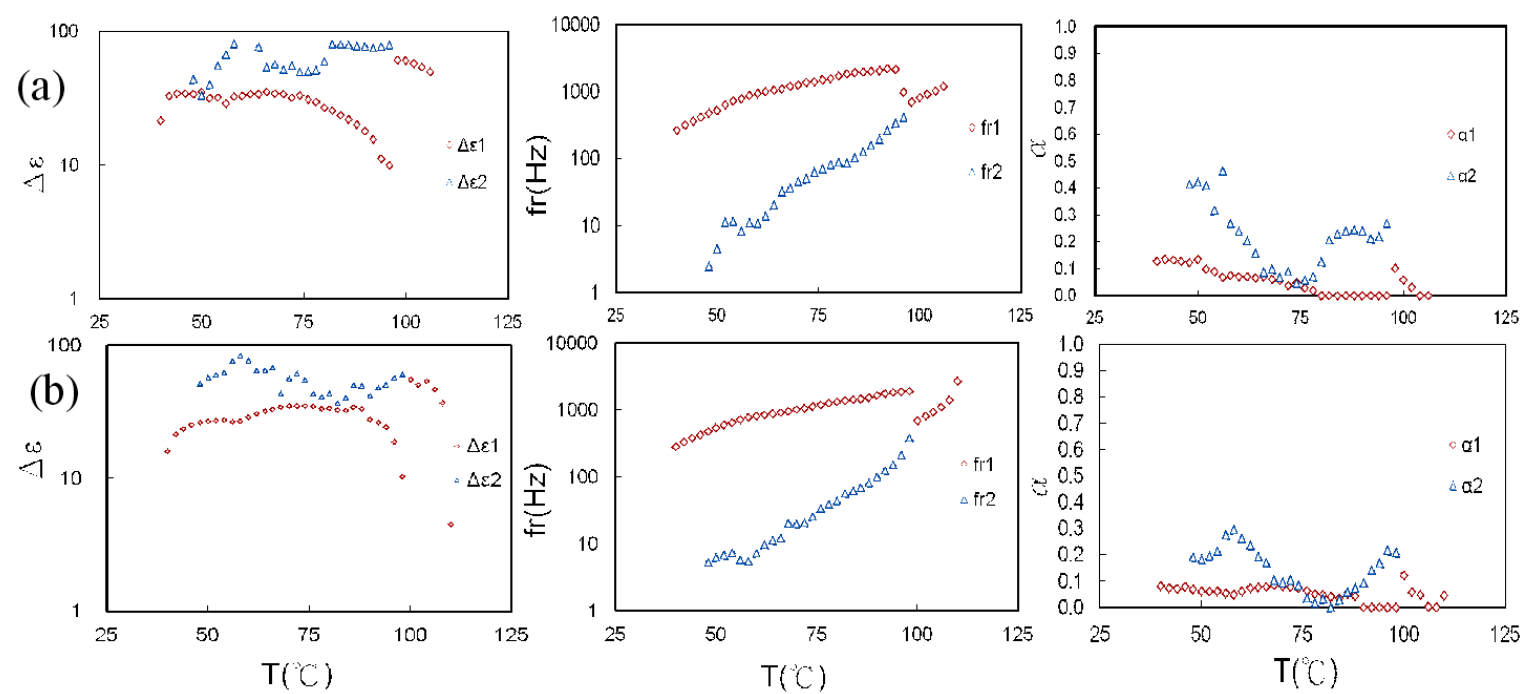

Fig. 5. Temperature dependence of dielectric strength $\Delta \varepsilon_{j}$, relaxation frequency $f_{r_{j}}$, and dispersion parameter $\alpha_{j}$ in

(a) sample A and (b) sample B ( $j=1$ for the mode at medium frequencies, and $j=2$ for the mode at low frequencies).

As seen in Fig. 5, for both samples, it is worth noting that a jump variation at the temperature dependence of $\Delta \varepsilon_{1}$ occurred in the phase transition from the $\mathrm{SmC}^{*}$ phase to the $\mathrm{SmC} \gamma^{*}$-like phase when cooling due to a strong contribution of the $\mathrm{SmC} \gamma^{*}$-like phase, which is associated to a strong contribution of the ferrielectric Goldstone mode [31]. Moreover, despite the jump variation, the relaxation frequency $f_{r_{j}}$ decreased with decrease of temperature, which was as reported in the $\mathrm{SmC} \gamma^{*}$ phase in Ref. [32]. This situation can be interpreted by $f_{\mathrm{r}} \propto p^{-2}$, where $p$ is the helical pitch. Since the helical pitch increased in the $\mathrm{SmC} \gamma^{*}$ phase in the cooling process [33], the relaxation frequency should decrease with decreasing temperatures [32]. In addition, for the two samples, the magnitudes of the obtained fitted parameters and their variations with temperature were similar, as shown in Fig. 5. The results imply that the difference between $(S, S)$-configuration and $(S, R)$-configuration in the diastereomeric structure had a weak effect on the dielectric variation for the FLC samples.

Comparing the two relaxation modes presented in the dielectric spectra of Fig. 5, the dielectric intensity of the specific relaxation mode in the $\mathrm{SmC} \gamma^{*}$-like phase was greater than that of the GM. However, in Ref. [28] (Sarmento et al.), the dielectric study in chiral liquid crystals showed that dielectric intensity for the specific relaxation mode in the ferrielectric phase $\left(\mathrm{SmC}_{\gamma}^{*}\right)$ was weaker than that for the GM. On the other hand, in Ref. [19], Hiller et al. have proposed that the relaxation strength of the ferrielectric process is very sensitive to the surface conditions. For example, Hollidt et al. have showed that the intensity of the ferrielectric Goldstone mode is approx- imately 5 times higher than that of the GM in thicker cells. Moreover, the molecular structures of the FLC samples in our study are different from those in Ref. [28]. Therefore, in our experiment, the phenomenon that the dielectric strength was stronger for the specific relaxation mode in the $\mathrm{SmC} \gamma^{*}$-like phase than that for the GM could be attributed to the specific molecular structures and surface conditions of the FLC samples.
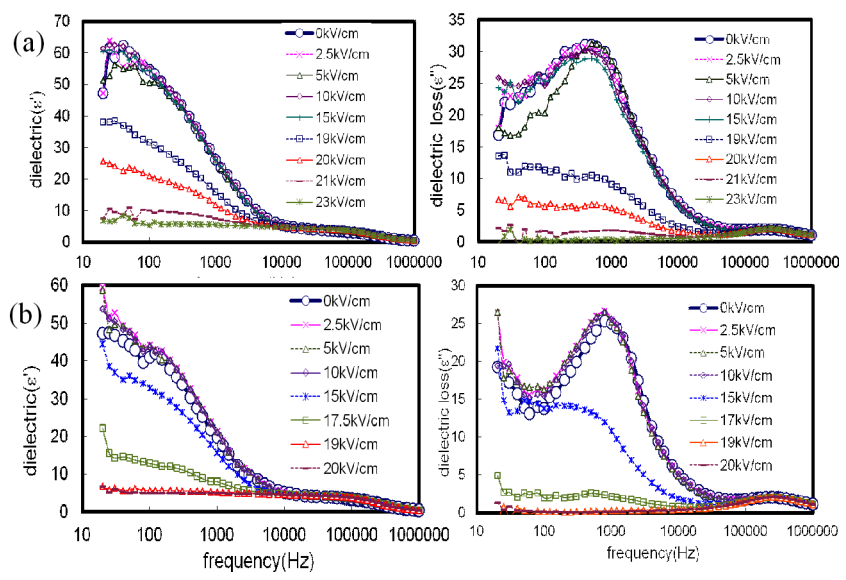

Fig. 6. Real part $\varepsilon^{\prime}$ and imaginary part $\varepsilon^{\prime \prime}$ of dielectric permittivity versus frequency under different bias electric fields of (a) sample A at $92^{\circ} \mathrm{C}$, (b) sample B at $102{ }^{\circ} \mathrm{C}$.

On the other hand, according to Ref. [34, 35], the intensity of GM is usually suppressed by a DC bias electric field. Figure 6 shows the bias effect on dielectric spectra for the two samples at certain temperatures. 
As shown in Fig. 6a, for sample A, whose diastereomeric structures was of $(S, S)$-configuration, the relaxations in the medium frequencies were systematically suppressed with increasing DC bias electric fields, which was attributed to the unwinding of the helical structure of the FLC molecules in homogeneous configuration [3638 . On the other hand, for sample B, whose diastereomeric structures is of $(S, R)$-configuration, Fig. $6 \mathrm{~b}$ shows a novel dielectric variation for the bias field effect. That is, the values of both the real part $\varepsilon^{\prime}$ and imaginary part $\varepsilon^{\prime \prime}$ of complex dielectric $\varepsilon^{*}$ increased with the increase of bias fields when the fields were below a specific strength. In contrast, the values of $\varepsilon^{\prime}$ and $\varepsilon^{\prime \prime}$ decreased with increasing fields when the bias fields were over a specific value. This particular dielectric variation corresponded to that in our previous study which have been published in Ref. [20]. To interpret the underlying mechanism of the enhancement of Goldstone mode, we have proposed a microscopic molecular orientation of the FLC samples with the $(S, R)$-configuration diastereomeric structures under the bias electric field in Ref. [20].

In order to get an understanding of the effect of the bias electric field on the low-frequency relaxation mode of the two samples in the $\mathrm{SmC} \gamma^{*}$-like phase. Figure 7a and $\mathrm{b}$ shows the dielectric spectra under various $\mathrm{DC}$ bias fields of the sample $\mathrm{A}$ at $82^{\circ} \mathrm{C}$ and sample $\mathrm{B}$ at $92^{\circ} \mathrm{C}$, respectively.
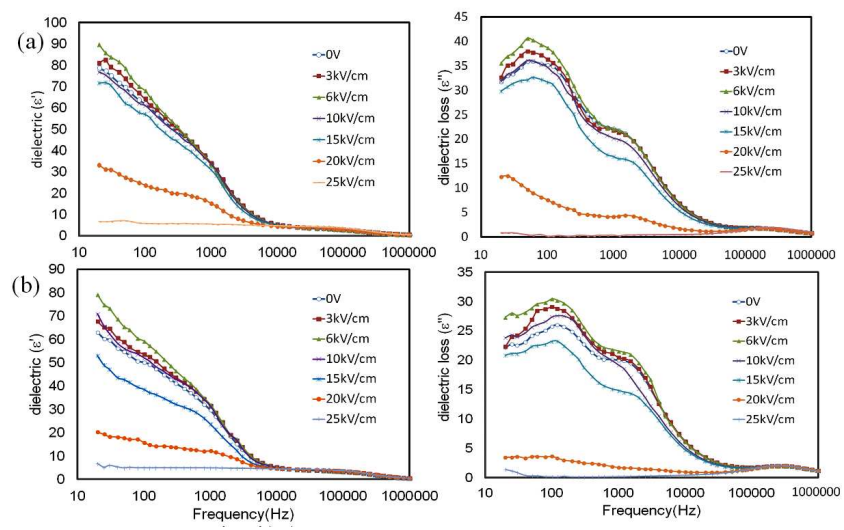

Fig. 7. Real part $\varepsilon^{\prime}$ and imaginary part $\varepsilon^{\prime \prime}$ of dielectric permittivity versus frequency under different bias electric fields of (a) sample A at $82^{\circ} \mathrm{C}$, (b) sample B at $92{ }^{\circ} \mathrm{C}$.

As shown in Fig. 7, with the increasing external bias field, the amplitude of the low-frequency relaxation process of both samples gradually increased in the beginning. When the bias field was above a specific value, the amplitude of relaxation process decreased with increasing bias fields. This was due to the electric-fieldinduced phase transition from $\mathrm{SmC}^{*}$ phase to the unwound $\mathrm{SmC}^{*}$ phase [39-41], which provided an evidence that the low-frequency relaxation mode can be identified as ferrielectric Goldstone mode. On the other hand, as shown in Fig. 7, under weak bias fields, the increasing variation of the medium-frequency relaxation of sample B is similar to that shown in Fig. 6. This could be explained by the microscopic molecular orientation of the FLC samples with the $(S, R)$-configuration diastereomeric structures under the bias electric field [20].

\section{Summary}

In conclusion, the spontaneous polarization and the molecular dynamics of two diastereomeric ferroelectric liquid crystals with a phenyl core structure were studied. The two types of diastereomeric structures were $(S, S)$ configuration and $(S, R)$-configuration chiral compounds. For the two samples, the values of spontaneous polarization and their variations with temperature were similar. The presence of the Goldstone mode in the ferroelectric phase $\left(\mathrm{SmC}^{*}\right)$ appeared in the complex dielectric spectra for the two samples. It is worth noting that the third relaxation mode implies that there existed a ferrielectriclike phase ( $\mathrm{SmC} \gamma^{*}$-like) for the two FLC samples. The dielectric strength of the specific mode that appeared in the $\mathrm{SmC} \gamma^{*}$-like phase was greater than that of the Goldstone mode, which must be attributed to the specific core structure of the FLC molecules. Moreover, under the DC bias fields on the dielectric spectra, two novel behaviors were observed. First, the Goldstone mode for the FLC samples of $(S, R)$-configuration structure was slightly enhanced by low DC bias fields. Second, the amplitude of low-frequency relaxation process of both samples increased with increasing bias fields when the fields were below a specific strength. In contrast, the amplitude of low-frequency relaxation process decreased with increasing fields when the bias fields were over a specific value. The results of this study depict the fundamentals of the molecular dynamics of FLCs in relation to their molecular structures, providing implications for device applications under alternating electromagnetic fields.

\section{Acknowledgments}

The authors would like to express sincere gratitude to Prof. Win-Lian Tsing of the National Kaohsiung Normal University Department of Chemistry for providing the FLC samples. The study was supported by the NSC 102-2120-S-002-006.

\section{References}

[1] J.W. Goodby, R. Blinc, N.A. Clark, S.T. Lagerwall, M.A. Osipov, S.A. Pikin, T. Sakurai, K. Yoshino, B. Zeks, in: Ferroelectric Liquid Crystals Principles, Properties, and Applications, Gordon and Breach Sci. Pub., Philadelphia 1991.

[2] S.K. Kundu, B.K. Chaudhuri, J. Appl. Phys. 94, 2271 (2003).

[3] T. Uemoto, K. Yoshino, Y. Inuishi, Jpn. J. Appl. Phys. 18, 1261 (1979).

[4] T.D. Ray, S. Kundu, P. Nayek, T.P. Majumder, S.K. Roy, W. Haase, Curr. Appl. Phys. 9, 605 (2009). 
[5] S.L. Wu, S. Senthil, Liq. Cryst. 31, 1573 (2004).

[6] W.L. Tsai, K.Y. Huang, C.Y. Hsueh, K.T. Wang, C.C. Wen, H.M. Lai, P.S. Cheng, J. Chin. Chem. Soc. 53, 1385 (2006).

[7] M. Marzec, W. Haase, E. Jakob, M. Pfeiffer, S. Wrobel, T. Geelhaar, Liq. Cryst. 14, 1967 (1993).

[8] A. Levstik, T. Carlsson, C. Filipic, I. Levstik, B. Zeks, Phys. Rev. A 35, 3527 (1987).

[9] L.A. Beresnev, M. Pfeiffer, S.A. Pikin, W. Haase, L.A. Blinov, Ferroelectrics 132, 99 (1992).

[10] S.K. Kundu, S. Yagihara, A. Yoshizawa, Liq. Crys. 34, 981 (2007)

[11] S. Hiller, L.A. Beresnev, S.A. Pikin, W. Haase, Ferroelectrics 180, 153 (1996).

[12] T. Carlsson, B. Zeks, C. Filipic, A. Levstik, Phys. Rev. A 42, 877 (1990).

[13] S.K. Kundu, B.K. Chaudhuri, L. Catala, S. Mery, Liq. Cryst. 29, 837 (2002).

[14] S. Merino, F. de Daran, M.R. de la Fuente, M.A.P. Jubindo, T. Sierra, Liq. Cryst. 23, 275 (1997).

[15] J.M. Czerwiec, R. Dabrowski, K. Garbat, M. Marzec, M. Tykarska, A. Wawrzyniak, S. Wrobel, Liq. Cryst. 39, 1503 (2012).

[16] P. Perkowski, M. Mrukiewicz, K. Garbat, M. Laska, U. Chodorow, W. Piecek, R. Dabrowski, J. Parka, Liq. Cryst. 39, 1237 (2012).

[17] P. Perkowski, M. Mrukiewicz, M. Zurowska, R. Dabrowski, L. Jaroszewicz, Liq. Cryst. 40, 864 (2013).

[18] M. Mrukiewicz, P. Perkowski, K. Garbat, R. Dabrowski, Liq. Cryst. 41, 1537 (2014).

[19] S. Hiller, S.A. Pikin, W. Haase, J.W. Goodby, I. Nishiyama, Jpn. J. Appl. Phys. 33, L1096 (1994).

[20] L.C. Huang, C.M. Fu, Phys. B 473, 54 (2015).

[21] W.L. Tsai, K.Y. Huang, C.Y. Hsueh, K.T. Wang, C.C. Wen, H.M. Lai, P.S. Cheng, J. Chin. Chem. Soc. 53, 1385 (2006).

[22] H.C. Lee, M.J. Hsie, S.W. Yeh, P.S. Cheng, W.L. Tsai, J. Chin. Chem. Soc. 49, 379 (2002).

[23] W.L. Tasi, S.W. Yeh, M.J. Hsie, H.C. Lee, Liq. Cryst. 29, 251 (2002).
[24] T. Datta, S. Kundu, P. Nayek, T.P. Majumder, S.K. Roy, W. Haase, Curr. Appl. Phys. 9, 605 (2009).

[25] A. Chelkowski, Dielectric Physics, Polish Scientific Publishers Polish Scientific Publishers \& Elsevier, New York 1980

[26] G.P. Sinha, F.M. Aliev, Phys. Rev. E 58, 2001 (1998).

[27] Relaxation Phenomena, Eds. S. Wrobel, W. Haase, Springer-Verlag, Berlin 2003.

[28] S. Sarmento, P.S. Carvalho, M.R. Chaves, F. Pinto, H.T. Nguyen, Liq. Cryst. 28, 673 (2001).

[29] P. Perkowski, Opto-Electron. Rev. 19, 176 (2011).

[30] G.W. Bak, M. Wojciechowski, M. Tykarska, J. Mol. Liq. 201, 43 (2015).

[31] M. Kaspar, P. Bilkova, A. Bubnov, V. Hamplova, V. Novotna, M. Glogarova, K. Knızek, D. Pociecha, Liq. Cryst. 35, 641 (2008).

[32] M. Wojciechowski, L.A. Gromiec, G.W. Bak, J. Mol. Liq. 124, 7 (2006).

[33] M. Cepic, E. Górecka, D. Pociecha, B. Zeks, H.T. Nguyen, J. Chem. Phys. 117, 1817 (2002).

[34] S.U. Vallerien, E. Kremer, H. Kapitza, R. Zentel, W. Frank, Phys. Lett. A 138, 219 (1989).

[35] D. Goswami, D. Sinha, A. Debnath, P.K. Mandal, S.K. Gupta, W. Haase, D. Ziobro, R. Dabrowski, J. Mol. Liq. 182, 95 (2013).

[36] S. Kumari, I.M.L. Das, R. Dabrowski, Physica B 407, 90 (2012).

[37] J. Hemine, A. Daoudi, C. Legrand, A El Kaaouachi, A. Nafidi, M. Ismaili, N. Isaert, H.T. Nguyen, Physica B 405, 2151 (2010).

[38] G. Singh, G. Vijaya Prakash, S. Kaur, A. Choudhary, A.M. Biradar, Physica B 403, 3316 (2008).

[39] C.J. Yu, J.H. Lee, S.D. Lee, H.T. Nguyen, Jap. J. Appl. Phys. 38, 5178 (1999).

[40] K.L. Sandhya, J.K. Song, Y.P. Panarin, J.K. Vij, S. Kumar, Phys. Rev. E 77, 051707 (2002).

[41] K. Hiraoka, A.D.L. Chandani, E. Gorecka, Y. Ouchi, H. Takezoe, A. Fukuda, Jap. J. Appl. Phys. 29 , L1473 (1990). 